Z. klin. Chem. u. klin. Biochem.

7. Jg., S. 521-524, September 1969

\title{
Ein einfacher dünnschichtchromatographischer Suchtest zur Erkennung von Hyperaminacidämien
}

\author{
Von F. Kraffczyk, R. Helger und H. Lang \\ Biochemiscbe Abteilung, E. Merck AG, Darmstadt
}

(Eingegangen am 5. Juni 1969)

Es wird ein einfacher dünnschichtchromatographischer Suchtest zur gleichzeitigen Erkennung aller wichtigen Hyperaminacidämien beschrieben. Plasma wird auf Cellulose-Fertigplatten mit dem Laufmittel Butanol/Aceton/Eisessig/Wasser (35:35:10:20, v/v) chromatographiert. Pathologische Konzentrationserhöhungen erkennt man beim Vergleich mit einem auf derselben Platte mitgeführten Standard. Zum Nachweis von Histidinämien verwendet man das Laufmittel tert. Butanol/Äthylmethylketon/25proz.Ammoniak/Diäthylamin/ Wasser $(50: 30: 10: 0,4: 20, \mathrm{v} / \mathrm{v})$.

Durch Verwendung eines fertigen Reagenziensatzes ist die Methode auch in solchen Laboratorien anwendbar, die keine Erfahrung in der Dünnschichtchromatographie haben.

\section{A simple thin layer chromatographic screening test for the detection of byperaminoacidaemias}

A simple TLC screening test for detection of all important types of hyperaminoacidaemia is presented. Plasma is chromatographed on precoated cellulose plates using the eluant butanol/acetone/acetic acid/water $(35: 35: 10: 20, \mathrm{v} / \mathrm{v})$. Pathological rises in the concentration of amino acids are detected by comparison with a standard chromatographed on the same plate. Histidinaemia is detected by chromatography with tert. butanol/ethylmethylketone/25\% ammonia/diethylamine/water (50:30:10:0.4:20, v/v). With the aid of a reagent set this method can also be used in laboratories which do not have previous experience in thin layer chromatography.

Zur Erkennung von Hyperaminacidämien dienen gegenwärtig mikrobiologische Tests (1) sowie chromatographische Methoden. Die Säulenchromatographie nach MOore und STEIN (2) liefert quantitative Ergebnisse für alle in Frage kommenden Aminosäuren, ist aber recht aufwendig. Für den Nachweis einer Anomalie genügen halbquantitative Verfahren, wie Papier- und Dünnschichtçhromatographie. Die bisher beschriebenen Verfahren (3-10) sind entweder verhältnismäßig zeitraubend, oder sie erfassen nur einige Aminosäuren, oder es mangelt ihnen an Nachweisempfindlichkeit, insbesondere für Phenylalanin.

Wir haben ein chromatographisches System entwickelt, das

1. eine einfache Durchführung des Tests erlaubt,

2. möglichst viele Hyperaminacidämien erfaßt,

3. eine ausreichende Nachweisempfindlichkeit für Phenylalanin aufweist.

Gute Trennungen über den ganzen in Frage kommenden Polaritätsbereich bei sehr kurzer Laufzeit' (30 Min. bei $7 \mathrm{~cm}$ Laufstrecke) erzielt man bei Zweifachentwicklung mit Aceton/Eisessig/Wasser (70:10:20 v/v) auf mikrokristalliner Cellulose (10). Ein Nachteil dieses Laufmittels ist die geringe Zonenschärfe in Frontnähe. Phenylalanin ist deshalb erst bei Konzentrationen über $5 \mathrm{mg} / 100 \mathrm{ml} \mathrm{zu}$ erkennen. $\mathrm{Da}$ die Normalwerte bei $2 \mathrm{mg} / 100 \mathrm{ml}$ (11) liegen und bei Neugeborenen im Alter von mehr als 4 Tagen Konzentrationen über $4 \mathrm{mg} / 100$ $\mathrm{m} l$ bereits als Verdachtsfälle gelten (12), eignet sich das Laufmittel nicht für einen Suchtest für Phenylketonurie bei Neugeborenen.

Auf Cellulose erhält man gute Trennungen bei sehr scharfen Banden mit dem Laufmittel Butanol/Eisessig/
Wasser (60:15:25 v/v). Für die Chromatographie der Plasmaaminosäuren eignet sich dieses System jedoch weniger, da die Trennung der Aminosäuren durch andere Plasmabestandteile gestört wird.

\section{Ergebnisse}

Wir fanden, daß eine ausgezeichnete Auftrennung der Plasmaaminosäuren über den ganzen Bereich bei hoher Bandenschärfe auf mikrokristalliner Cellulose mit der mobilen Phase Butanol/Aceton/Eisessig/Wasser (35:35: $10: 20 \mathrm{v} / \mathrm{v}$ ) durch Zweifachentwicklung erreicht wird. Die Aminosäuren sind in diesem System über die ganze Laufstrecke verteilt (Abb. 1). Das im Plasma enthaltene Eiweiß bleibt am Start zurück. Die Ausführung der Chromatographie der Plasmaaminosäuren und die Auswertung der Chromatogramme ist relativ einfach. Eine besonders hohe Nachweisempfindlichkeit des Phenylalanins $(2,5 \mathrm{mg} / 100 \mathrm{~m} l)$ wird durch die Verwendung spezieller Celluloseplatten mit einer Schichtdicke von $80-100 \mu \mathrm{m}$ erreicht (1).

Bei der Durchführung des Tests wird nach Shriver (4) Blut in heparinisierten Kapillaren aufgenommen und durch Zentrifugieren Plasma gewonnen. Auf eine Platte werden verschiedene Plasmaproben sowie ein geeigneter Standard aufgetragen. Man benutzt dazu Einmal-Mikrokapillaren. Ein Auftragegerät erleichtert das für gute Trennungen vorteilhafte strichförmige Auftragen definierter Lösungsmengen. Die Chromatographie in dem beschriebenen System dauert etwa $90 \mathrm{Min}$. bei Zweifachentwicklung. Das Anfärben der Aminosäuren erfolgt mit Ninhydrin. Danach wird die Platte mit einer Modifikation von EHRLICH's Reagenz behandelt, wobei Prolin und Hydroxyprolin als rote Banden erscheinen, 


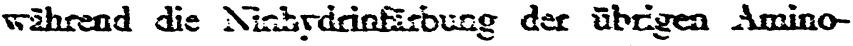
siuren verblaßt. Die Auswertiag eriolgt durch risuellec. Vergleich ait dexi Standardgemisch. Die Lokalisation der i-irirosiuren in den einzelser Bacces zeigt Abbildung 1.

Treten eine oder mehrere birden eine Plasmajproje stärker jerro: als die enteprecinenden Bärden des

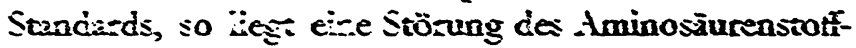
weciseis roz. In Abbildung 1 sird nur die im nosmalen

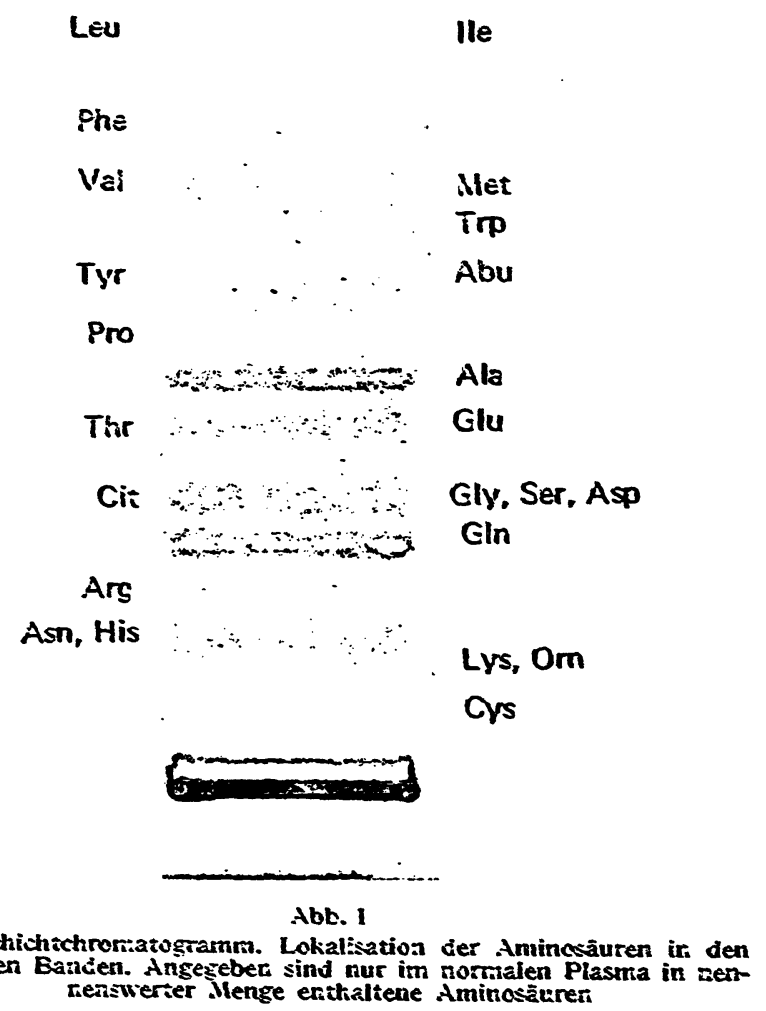

Plasma in newneaswerter Menge enthaltenen Aminosāuren aưfgeführt. In pathologischen Fällen lönnen auch noch weitere Aminosāuren auftreten, deren Lagen im Chromatogramm aus Abbildung 2 zu entnehmen sind. Die absoluten Steighöhen sind schwer reproduzierbar, jedoch bleibt die Reihenfolge der tminosāuren stets erhalten. Die Empfindlichkeit des Tests ist nicht für alle Aminosāuren gleich. Grenzkonzentrationen, die mit Sicherheit als Erhöhung wahrgenommen werden, sind aus Abbildung 2 exsichtlich. Im Standard fehlen wegen mangelader Haltbarkeit bzw. Löslichkeit dié Aminosāuren Glummin, Crstin und Omithin. Man orientiert sich hinsichtlich der Menge ron Glutamin und Crstin an den benachbarten normalen Plasmaproben. Das Fehlen ron Ornithin wird durch einen erhöhten Gehalt des Standards an Irsin ausgeglichen. Eine Verstärkung der Glycin/Serin-Bande beruht meist auf einer harmlosen

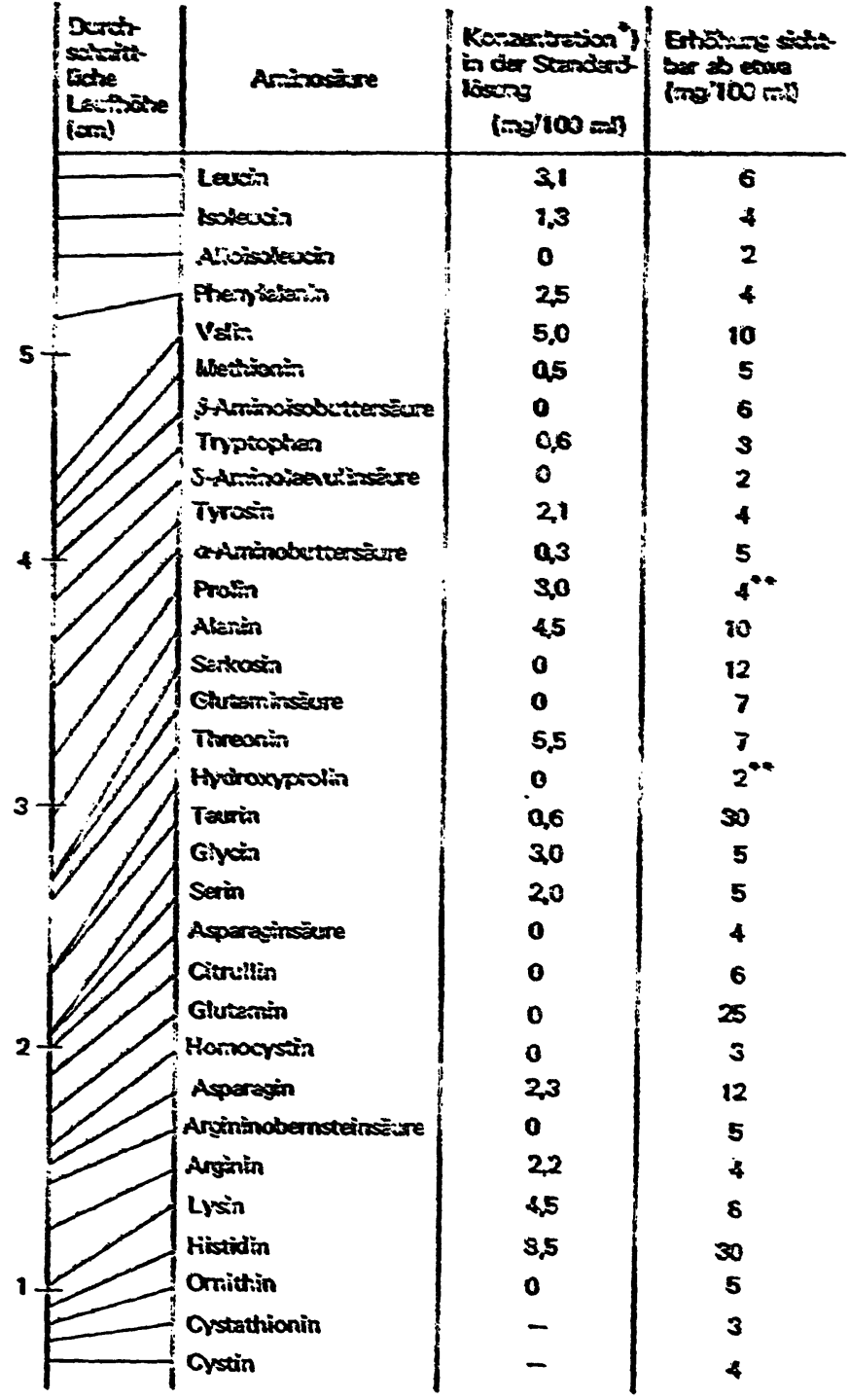

Abb. 2

Durchschnitiiidte Laufstrecke vor Aninosiuren, die in pathologischen Fällen auftreten kôrrien, izn Dünnschict:tctromatugramm (Entwicklung zweifach, Front bei $7,5 \mathrm{~cm}$ )

* Der Standard ezgibe ein Chromatogramm, das visuell etura dem Chromatogramm eines Plastras mit Amiriosäurenkonzertirationen im oberen Nomaibereich 5 Tage alter Neukgeborener gleictit Die einzelnen Werte sind richt ats Normaluerte ar verstehen

** Nach Reaktion mit dern Dimeshytaminobenzaldehyd/Phosphorșüure-Reagenz

Erhöhung der Serinkonzentration. Will man die äußerst seltenen Fälle ron Glỵcinàmie miterfassen, muß das betreffende Plasma nochmals auf eine Platte aufgetragen und durch Dreifach- oder Mietfach-Entwicklung getrennt werden. Dabei spaltet sich die Glrcin'SerinBande auf. Die obere Zone entspricht dem Glycin, die untere dem Serin. Anstelle des in der Testpackung 1) entbaltenen Laufmittels kann zur Auftrennung ron SerinjGlycin sowie zur Abtrennung ron Histidin das Laufmittel tert.-Bucanoly Athrimethrlketonj25 proz. AtmmoniakfDiäthylamin/Tasser $\quad(50: 30: 10: 0,4: 20$, v!v) rerwendet werden. Dieses Gemisch ist für die genannten Trennungen besser geeignet (13).

\section{Diskussion}

Die in Abbildung 2 in der letzten Spalte aufgeführten Konzentrationen sind Grenzlionzentrationen, ab denen 


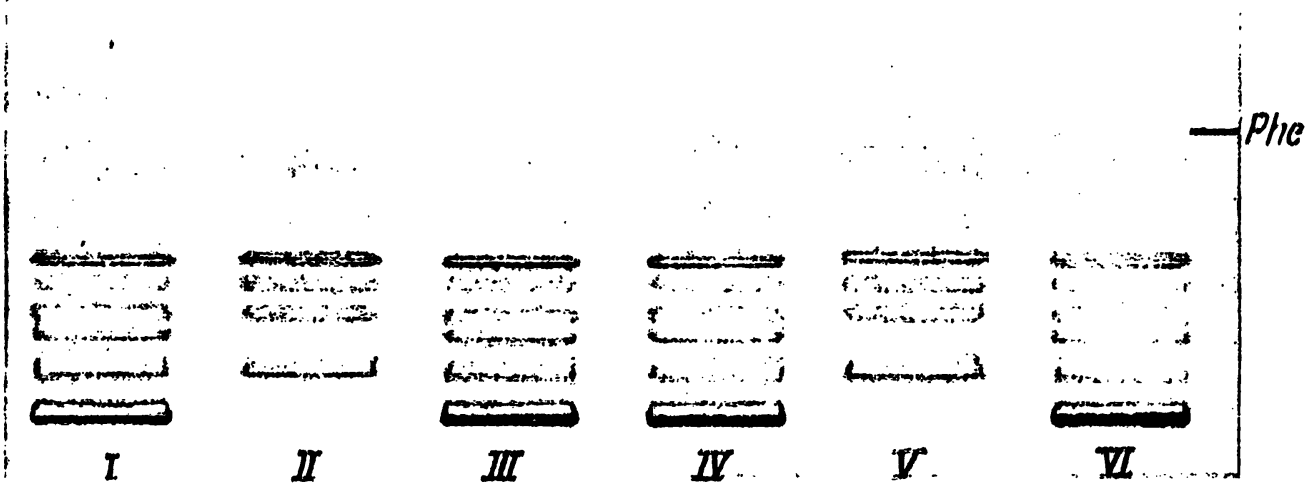

Ahli, :

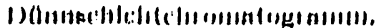

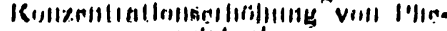
liyliltrilli.

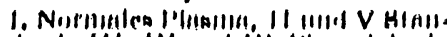

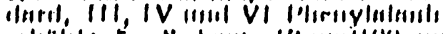

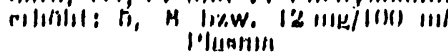

A1)1, 4

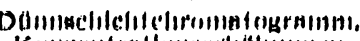

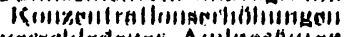
vorectiloderier Airlingadilion.

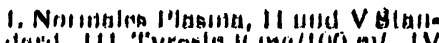

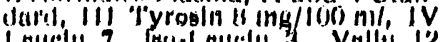

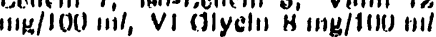

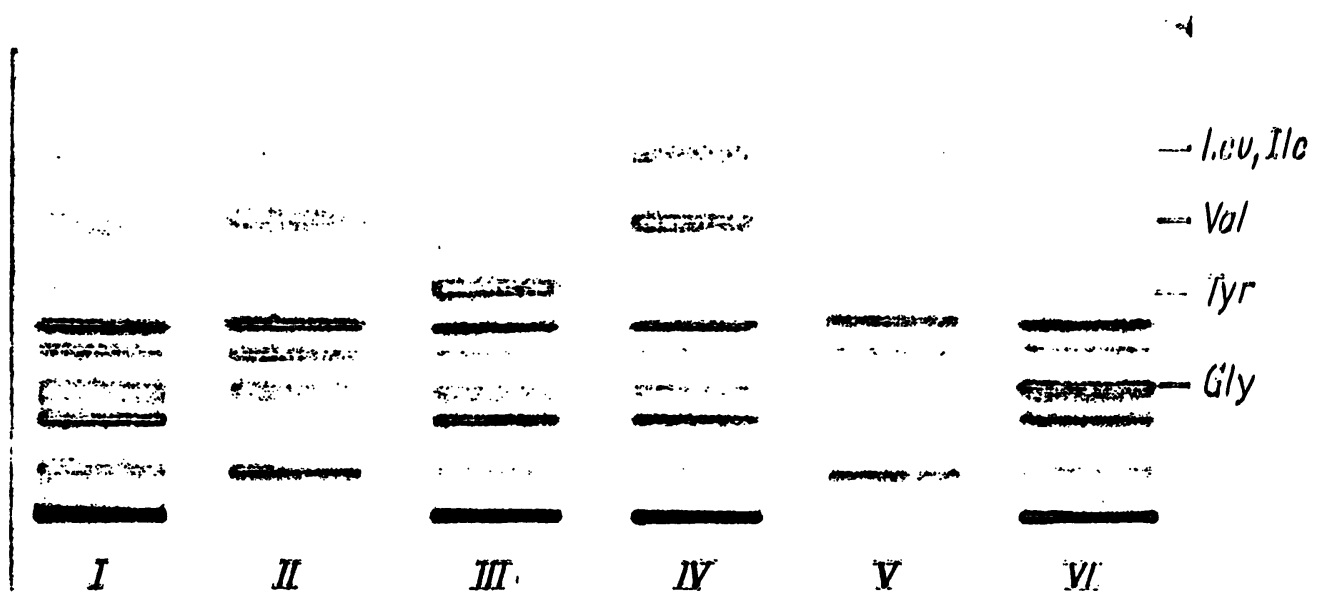

eine Panmabande gegendiber dem Standard veruteltet cryclicint. Dic absoluten Nach weitigrenken liegen wetientJich nledriger, \% B. flie Phenylatanin hei $2,5 \mathrm{mg} / 100 \mathrm{~m} /$. Dic Konyentrationen in der Standarellesiung, wurden vo gewählt, dal3 elnertielts nach Möglichkeit bei allen in Prage kommenden $\Lambda$ minoneidhmien die entepprechender Aminobituelsanden gegentiber dent Stafidard als verstiflet crsclieinen, andererseitn inbgliehnt wenig falseh positive Hrgebnisse crhalten werden. Dic Standard= IBrung liegt dewhalh deutlich fiber: den mittleren Planmakonzentrationen. Dle Abbildungen 3 und 4 \%elgen Shromatogramme mit Konzentrationserhohungen cin\%glner Aminosturen. Wlehtig ist, dal3 Phenylalanis bel Platuma. Jersm\%entrationen ab $4 \mathrm{mg} / 100 \mathrm{ml}$ eine deutliche Ver-

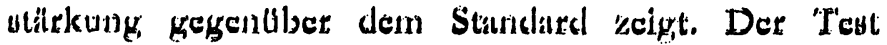
jot deshall, auch als Suchtest auf lhenyllectontrie in der Neugaborencuperiode gecignet. Die Mathode vereluigl hohe Nachweisemplindlichlecit im Palle eles l'benyl. alanims mit suter $\Lambda$ aftremnung liber den genanten Jolarithtelsereich. Daraus ergibt sich cine breite $\Lambda_{\text {n- }}$ wandbarkeit. Nack dem Hegebnis ciner Profung lin versehiedenen Jaboratorien sind mit dem 'f'ent folgende Anomallen erkenmbar. (13): Phenylketomuric und I lyper.

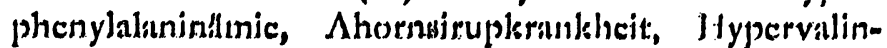
thmic, Hypertnethioninitmic (be) JJomocystinturic wnd

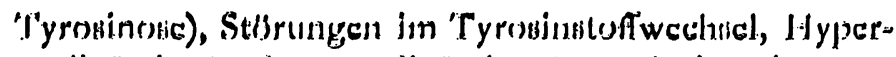

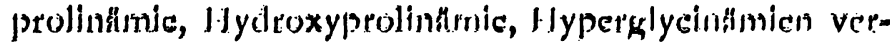
Hehiedener Genese, Jyperlynimilmich, Orrithinitmic und - bei hoblieren Konzentratlonen brow. Hacl Variation des I Aufunittels $=$ Jistidinämien.

Der 'Test erfordert keinen apparativen Aulwand und ind

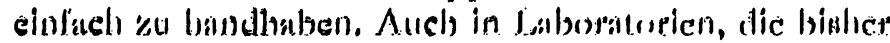

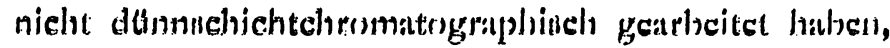

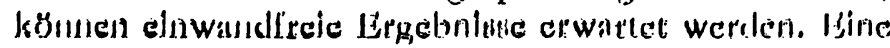

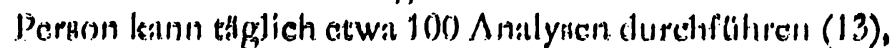

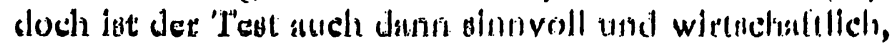
wenn tagjel nur 4-8 Analyaen dureligefilihrt werelen.

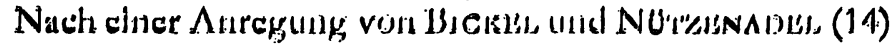
kann der. 'l'cat' auch \%ur Unterbuchung von 13lu1: ver-

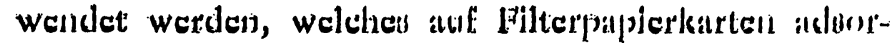

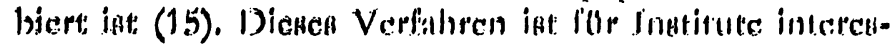
bant, die deratig gasimmalte Blutproben yur Unterauchung mit dem Guthrietest \%ugentudt erhaltes. Dic 13lutplittehen werden extrahiert und die Lxtrakte puinktformig aul dic 1)

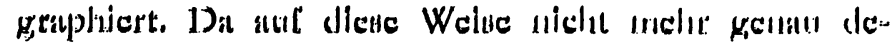

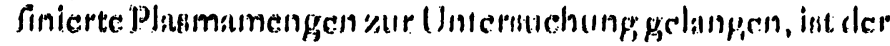

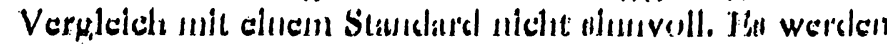

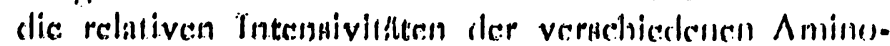

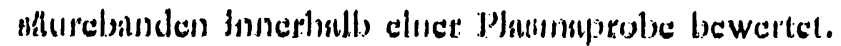




\section{Methodik}

\section{Reagenzien ${ }^{\mathbf{1}}$ )}

Sorptionsscbicbt

Cellulose-Fertigplatten

\section{Laufniittel}

n-Butanol/Aceton/Eisessig/Wasser $(35: 35: 10: 20, v / v)$. Das fertige Laufmittel ist bei Raumtemperatur 12 Stdn. haltbar.

Ninbydrin-Lösung

$0,4 \mathrm{M}$ Ninhydrin in $\mathrm{n}$-Butanol/Aceton $(50: 50, \mathrm{v} / \mathrm{v})$. Die Lösung ist im Kühlschrank mindestens drei Monate haltbar.

\section{Prolinreagenz}

$0,057 \mathrm{M} p$-Dimethylamino-benzaldehyd in Essigsäureäthylester/ 85 proz.Phosphorsäure/Eisessig/Wasser (50:6:34:10, v/v). Die Lösung ist bei Raumtemperatur mindestens 14 Tage haltbar.

\section{DC der Aminosäuren in Heparin-Plasma}

\section{Plasma}

Man füllt eine heparinisierte Kapillare mit Blut, schmilzt am blutfreien Ende zu, zentrifugiert und zerschneidet an der Trennlinie zwischen Blutkuchen und Plasma.

\section{Auftragen}

Je $2 \mu l$ Plasma bzw. Standard werden mit dem Auftragegerät ${ }^{2}$ ) und Mikrokapillare entsprechend Abbildung 5 aufgetragen.

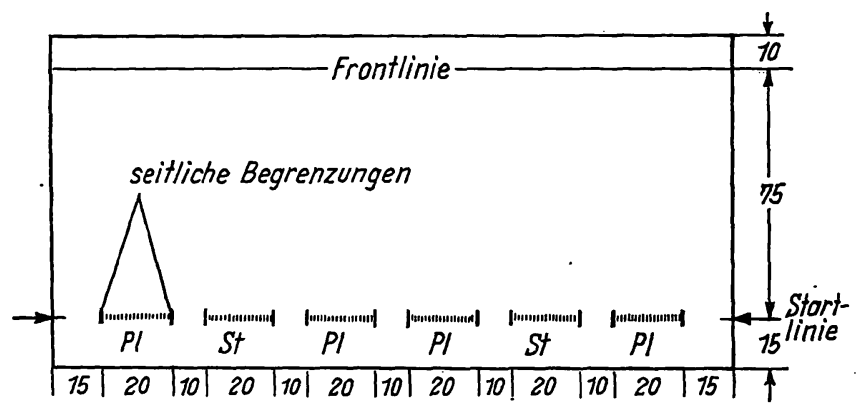

Abb. 5

Schema für das Auftragen von Standard-(St)- und Plasma-(PI)-Proben auf die DC-Platte $(200 \times 100)$. Alle Zahlenangaben in $\mathrm{mm}$

1) Alle Reagenzien sind in dem Reagenziensatz Merckotest $D C$ Aminosäuren im Plasma, Art. Nr. 3345 enthalten.

2) Das Auftragegerät ist in der Grundausstattung für Merckotest DC, Art. Nr. 3346 enthalten.

\section{Entvicklung und Nachweis}

Man entwickelt einmal bis zur Frontlinie (etwa $50 \mathrm{Min}$., keine Kammersättigung!) und trocknet mit dem Heizlüfter. Dann pipettiert $\operatorname{man} 3 \mathrm{ml}$ Ninhydrinlösung in die Kammer zu dem Laufmittel der ersten Entwicklung und mischt. Die getrocknete Platte wird in dieser Mischung erneut bis zur Frontlinie entwickelt. Zunächst wird mit dem Heizlüfter und anschließend 5 Min. im Trockenschrank bei $80^{\circ}$ getrocknet. Die Aminosäuren mit Ausnahme von Prolin und Hydroxyprolin erscheinen àls blaue Zonen. Zur Auswertung wird die Platte 5 Min. nach Entnahme aus dem Trockenschrank im Auflicht und im Durchlicht betrachtet.

Nachweis von Prolin und Hydroxyprolin: Ein Pinsel wird mit dem Prolinreagenz so getränkt, daß er gerade nicht tropft. Man bestreicht die bereits mit Ninhydrin behandelte Platte mit einem Pinselstrich parällel zur Lösungsmittelfront in Höhe der Alaninund Glycin-Zone (vgl. Abb. 1). Die Platte wird drei Minuten lang im Trockenschrank auf $110^{\circ}$ erhitzt. Prolin und Hydroxyprolin werden als rote Zonen sichtbar, falls die Konzentrationen dieser Aminosäuren hoch genug sind. Pathologische Erhöhungen in Plasmaproben sind in jedem Fall gut erkennbar. Die Farben verblassen nach einigen Minuten.

DC der Aminosäuren im Serumbei Blutabnahme mit Filterpapierkarten:

\section{Extraktion}

Aus den Filterpapierkarten wird ein blutgettänktes Blättchen von $1 \mathrm{~cm}$ Durchmesser ausgestanzt oder, ausgeschnitten. Das Blättchen wird auf den Boden eines verschließbaren Gefäßes etwa gleichen Bodendurchmessers gelegt (z. B. eine 5-m/-Flasche). Dazu werden $0,1 \mathrm{ml}$ A thanol/Wasser $(70: 30, \nabla / \mathrm{v})$ pipettiert. Es ist darauf zu achten, daß das Extraktionsmittel das Blättchen vollständig bedeckt. Eventuell ist die Lage des Blättchens mit dem Spatel zu korrigieren. Das Gefäß wird verschlossen und 12 bis 24 Stdn. bei Raumtemperatur stehengelassen.

\section{Auftragen}

Die Gefäße mit der Extraktionslösung werden kurz umgeschüttelt. Dann trägt man je $2 \mu l$ als möglichst kleinen Punkt im Abstand von je $1 \mathrm{~cm}$ auf die Startlinie auf. Auf diese Weise gelingt es, 18 Proben auf einer Platte unterzubringen. Standard wird nicht aufgetragen.

\section{Entwicklung, Nacbweis und Beurteilung}

Entwicklung und Nachweis erfolgen wie beschrieben. Das Verhältnis von Fleckengrößen bzw. Intensitäteñ der einzelnen Aminosäurenzonen einer Serumprobe zueinander ist bei allen normalen Seren gleich. Wird beim Vergleich mit einem normalen Serum eine Verschiebung dieses Verhältnisses sichtbar, liegt der Verdacht einer Aminosäurenanomalie nahe.

Herrn ERKENS danken wir für die umsichtige Ausführung der Versuche.

\section{Literatur}

1. Guthrie, R., Birth Defects Original Article Series IV (No 6), Nov. 1968, pub. National Foundation - March of Dimes, S. 92-98. 2. Stern, W. H. und S. Moore, J. biol. Chemistry 211, 915 (1954). - 3. Efron, D. Young, H. W. Moser und R. A. McCready, New Engl. J. Med. 270, 1378 (1964). - 4. Scriver, C. R., E. Davies und A. M. Cullen, Lancet London 1964/II, 230. - 5. Cawley, L. P., W. L. Goodwin und P. Dibbern, Amer. J. Clin. Path. 48, 405 (1967). - 6. Ditrmann, J., Arch. Kinderbk. 177, 6 (1968). - 7. NirschKÉ, E., diese 'Z. 6, 208 (1968). - 8. Mellon, J. P. und A. G. Strven, J. Med. Laborat.
Tech. 23, 204 (1966). - 9. Meyer, J:, K. Droll und V. KLINGMÜLLER, Clin. chim. Acta.Amsterdam 18, 69 (1967). - 10. BLEN Nemann, H., Dtsch. Med. Wschr. 93, 1566 (1968). - 11. Hsia, D.Y.Y., J.L. Berman und M. M. Slatis, J. Amer. Med. Ass. 188 , 203 (1964). - 12. BrckeL, M., Dtsch. Arzteblatt 62, 717 (1965). 13. Bremer, $H$. J., W. Nützenader und $H$. Bickél, Mschr. Kinderh. 177, 32 (1969). - 14. BrCKel, M. und W. NütżENAdeI persönliche Mitteilung. - 15. Plozchr, E., Clin. chim. Acta Amsterdam 21, 271 (1968).
Dr. H. Lang

E. Merck AG.

61 Darmstadt 2

Postfach 4119 